\title{
Symmetry Properties of Optimal Relative Orbit Trajectories
}

\author{
Mauro Pontani \\ University “La Sapienza”, Via Salaria 851, 00138 Rome, Italy \\ Correspondence should be addressed to Mauro Pontani; mauro.pontani@uniromal.it
}

Received 14 December 2014; Revised 29 April 2015; Accepted 30 April 2015

Academic Editor: Juan F. San-Juan

Copyright (C) 2015 Mauro Pontani. This is an open access article distributed under the Creative Commons Attribution License, which permits unrestricted use, distribution, and reproduction in any medium, provided the original work is properly cited.

\begin{abstract}
The determination of minimum-fuel or minimum-time relative orbit trajectories represents a classical topic in astrodynamics. This work illustrates some symmetry properties that hold for optimal relative paths and can considerably simplify their determination. The existence of symmetry properties is demonstrated in the presence of certain boundary conditions for the problems of interest, described by the linear Euler-Hill-Clohessy-Wiltshire equations of relative motion. With regard to minimum-fuel paths, the primer vector theory predicts the existence of several powered phases, divided by coast arcs. In general, the optimal thrust sequence and duration depend on the time evolution of the switching function. In contrast, a minimum-time trajectory is composed of a single continuous-thrust phase. The first symmetry property concerns minimum-fuel and minimum-time orbit paths, both in two and in three dimensions. The second symmetry property regards minimum-fuel relative trajectories. Several examples illustrate the usefulness of these properties in determining minimum-time and minimum-fuel relative paths.
\end{abstract}

\section{Introduction}

Minimum-time and minimum-propellant-consumption space trajectories have represented a subject of great relevance in astrodynamics since the 1950s. Pioneering works in this field are due to Lawden [1], Leitmann [2], Miele [3], and Edelbaum [4]. More recently, Marec [5], Vinh [6], and Prussing $[7,8]$ provided relevant contributions, with reference to different types of aerospace missions.

Specifically, relative orbital motion has attracted an increasing interest in the last decades, both for formation flying design and for the purpose of planning spacecraft proximity maneuvers. A very useful mathematical framework for investigating relative orbital motion is represented by the Euler-Hill-Clohessy-Wiltshire (EHCW) equations [9]. They derive from the linear expansion of the dynamics equations that govern position and velocity of a spacecraft relative to a real or virtual vehicle, placed in a neighboring reference circular orbit. In the 1960s Tschauner and Hempel [10] addressed the problem of relative orbital motion with respect to a reference elliptic orbit, whereas Abrahamson and Stern [11] provided an extension to hyperbolic paths. Further works $[12,13]$ have been devoted to investigating relative orbital motion involving eccentric orbits, through the use of either the true or the eccentric anomaly as the independent variable. Most recently, Melton [14] developed an approximate solution that depends explicitly on time and uses a series expansion in terms of the reference orbit eccentricity. Schaub [15, 16] employed orbit element differences relative to a chief orbit with the intent of describing the relative orbit geometry and in order to establish relative orbits invariant with respect to the Earth oblateness. The coordinated motion of a set of spacecraft is a crucial issue for formation flying, and several works [17-23] have been focused on this central topic, with the final aim of minimizing the control effort needed for relative configuration maintenance. However, orbital maneuvers may be needed both for formation flying disposal and, more generally, in the context of proximity maneuvers aimed at rendezvous or orbit rephasing. Minimizing the fuel consumption leads to extending the satellite operational life or to enhancing its operational capabilities. As a pioneering contribution in this field, Prussing $[7,8]$ concentrated on applying the primer vector theory to two-, three-, and four-impulse relative orbit maneuvers. Carter [24] considered finite-thrust paths, by employing an interesting geometric approach similar to that adopted by Prussing. More recent contributions either deal 
with constrained impulsive and finite-thrust maneuvers [2529] or are focused on formation flying reconfiguration [30].

In general, the problem of spacecraft trajectory optimization is to be formulated as an optimal control problem, which consists in finding the optimal thrust sequence, magnitude, and direction that minimize propellant consumption or time of flight. Only a few simplified problems are amenable to a closed-form solution, and therefore numerical techniques are usually employed in trajectory optimization problems. Classical (indirect and direct) methods need a first-attempt approximate guess to yield a refined solution, and this can be challenging to provide. A recently introduced methodology, termed indirect heuristic technique [31], employs the necessary conditions for optimality, that is, the Pontryagin minimum principle, the Euler-Lagrange equations, and the Weierstrass-Erdmann conditions, in conjunction with a heuristic optimizer. The latter requires the conversion of the optimal control problem into a parameter optimization problem in order to yield the desired solution. This goal is achieved by employing the necessary conditions for optimality, which allow expressing the control variables as functions of the adjoint variables conjugate to the dynamics equations. This circumstance has the consequence that the initial values of the adjoint variables are treated as the unknown parameters sought by the heuristic solver. In this context, a particular selection of values of the unknown parameters is associated with an individual. A population of individuals, whose number increases as the number of unknown parameters increases, is generated and evolves toward the optimal parameter set. Some recently published papers $[32,33]$ prove the effectiveness and accuracy of this approach, used also for the illustrative examples reported in this work.

This paper has the purpose of illustrating a pair of symmetry properties that hold for optimal relative space trajectories and can simplify their determination. In the mathematical framework established by the EHCW equations, this work is intended to

(a) derive the analytical conditions related to minimumfuel and minimum-time relative trajectories in a unified perspective,

(b) prove a first symmetry property that holds for both minimum-fuel and minimum-time relative paths,

(c) report the statement and proof of a second symmetry property related to the optimal thrust sequence and direction of minimum-fuel relative trajectories,

(d) illustrate the use of both properties, also pointing out their specific utility.

The first symmetry property was employed in [32], where only feasibility of symmetric paths was proven, but not their optimality. The second symmetry property was demonstrated and employed in [33] and is reported in this paper for the sake of completeness. What will be proven is that both properties lead to doubtless computational advantages.

\section{Linear Orbit Theory}

The relative motion of a spacecraft with respect to a virtual or real target vehicle, placed in a neighboring reference orbit, can be described using linear orbit theory [9], which provides the equations of relative motion, referred to as the Euler-HillClohessy-Wiltshire (EHCW) equations.

The EHCW equations represent the linear expansion of the dynamics equations that govern position and velocity of the pursuing spacecraft $(P)$ relative to the target vehicle $(T)$, which is placed in a reference circular orbit of radius $R_{T}$. If $\mu$ is the gravitational parameter of the attracting body, the mean motion of $T$ (in this case equal to its constant angular rate) is $\omega=\sqrt{\mu / R_{T}^{3}}$. Let $\mathbf{r}_{i}$ and $\mathbf{v}_{i}$ represent the inertial position and velocity vectors (with $i=T$ or $P$ ) and $\rho$ the relative position of $P$. The EHCW equations are written in terms of the projections of $\boldsymbol{\rho}$ along the right-hand reference frame $\left(\widehat{r}_{T}, \widehat{\theta}_{T}, \widehat{h}_{T}\right)$, where $\widehat{r}_{T}$ and $\widehat{h}_{T}$ are, respectively, the unit vectors associated with the target position vector $\mathbf{r}_{T}$ and the specific angular momentum $\mathbf{h}_{T}\left(:=\mathbf{r}_{T} \times \mathbf{v}_{T}\right): \boldsymbol{\rho}:=\mathbf{r}_{P}-\mathbf{r}_{T}=$ $x \widehat{r}_{T}+y \widehat{\theta}_{T}+z \widehat{h}_{T}$. The EHCW equations are [9]

$$
\begin{aligned}
\ddot{x}-2 \omega \dot{y}-3 \omega^{2} x & =a_{x}, \\
\ddot{y}+2 \omega \dot{x} & =a_{y}, \\
\ddot{z}+\omega^{2} z & =a_{z},
\end{aligned}
$$

where $\mathbf{a}(t):=a_{x}(t) \widehat{r}_{T}+a_{y}(t) \widehat{\theta}_{T}+a_{z}(t) \widehat{h}_{T}$ can be regarded as a perturbing or thrust acceleration. Letting $\widetilde{\mathbf{x}}=$ $\left[\begin{array}{llllll}x_{1} & x_{2} & x_{3} & x_{4} & x_{5} & x_{6}\end{array}\right]^{T}=\left[\begin{array}{llllll}x & y & z & \dot{x} & \dot{y} & \dot{z}\end{array}\right]^{T},(1)-(3)$ can be rewritten in the form of state equations as

$$
\dot{\widetilde{\mathbf{x}}}(t)=A \widetilde{\mathbf{x}}(t)+\widetilde{\mathbf{a}}(t),
$$

where

$$
\begin{aligned}
A & =\left[\begin{array}{cccccc}
0 & 0 & 0 & 1 & 0 & 0 \\
0 & 0 & 0 & 0 & 1 & 0 \\
0 & 0 & 0 & 0 & 0 & 1 \\
3 \omega^{2} & 0 & 0 & 0 & 2 \omega & 0 \\
0 & 0 & 0 & -2 \omega & 0 & 0 \\
0 & 0 & -\omega^{2} & 0 & 0 & 0
\end{array}\right], \\
\widetilde{\mathbf{a}}(t) & =\left[\begin{array}{c}
0 \\
0 \\
0 \\
a_{x}(t) \\
a_{y}(t) \\
a_{z}(t)
\end{array}\right] .
\end{aligned}
$$


Vector equation (4) is equivalent to six linear differential equations with constant coefficients and admits a general solution composed of two terms

$$
\widetilde{\mathbf{x}}(t)=\Theta\left(t, t_{0}\right) \widetilde{\mathbf{x}}_{0}+\int_{t_{0}}^{t} \Theta(t, \tau) \widetilde{\mathbf{a}}(\tau) d \tau,
$$

$$
\Theta(\tau)=\left[\begin{array}{ccc}
4-3 c(\omega \tau) & 0 & 0 \\
6 s(\omega \tau)-6 \omega \tau & 1 & 0 \\
0 & 0 & \cos (\omega \tau) \\
3 \omega s(\omega \tau) & 0 & 0 \\
6 \omega[c(\omega \tau)-1] & 0 & 0 \\
0 & 0 & -\omega s(\omega \tau)
\end{array}\right.
$$

where $s():=\sin ()$ and $c():=\cos ()$.

The first term of (6) is the general solution of the homogeneous system associated with (6); that is, it is the solution of the EHCW equations in the absence of $\mathbf{a}(t)$. If the initial time $t_{0}$ is set to 0 , it is relatively straightforward to show that-omitting the velocity components for the sake of brevity-the homogeneous term (denoted by the subscript " $h$ ") can be conveniently rewritten as

$$
\begin{aligned}
& x_{1, h}(t)=c_{1} \cos (-\omega t+\zeta)+\frac{2 c_{2}}{\omega}, \\
& x_{2, h}(t)=2 c_{1} \sin (-\omega t+\zeta)-3 c_{2} t+c_{3}, \\
& x_{3, h}(t)=c_{4} \cos (-\omega t+\xi),
\end{aligned}
$$

where the constants $\left\{c_{j}\right\}_{j=1, \ldots, 4}$ and the angles $\zeta$ and $\xi$ depend on the initial conditions $\left(x_{0}, y_{0}, z_{0}, \dot{x}_{0}, \dot{y}_{0}, \dot{z}_{0}\right)$ and have the following expressions:

$$
\begin{aligned}
c_{1} & =\sqrt{\left(3 x_{0}+\frac{2 \dot{y}_{0}}{\omega}\right)^{2}+\left(\frac{\dot{x}_{0}}{\omega}\right)^{2}}, \\
\sin \zeta & =\frac{\dot{x}_{0}}{\omega c_{1}}, \\
\cos \zeta & =\left(3 x_{0}+\frac{2 \dot{y}_{0}}{\omega}\right) \frac{1}{c_{1}}, \\
c_{2} & =2 \omega x_{0}+\dot{y}_{0}, \\
c_{3} & =y_{0}-\frac{2 \dot{x}_{0}}{\omega}, \\
c_{4} & =\sqrt{z_{0}^{2}+\left(\frac{\dot{z}_{0}}{\omega}\right)^{2}},
\end{aligned}
$$

where $\Theta$ is the state transition matrix, which obeys the following equation:

$$
\dot{\Theta}\left(t, t_{0}\right)=A \Theta\left(t, t_{0}\right) \quad \text { with } \Theta\left(t_{0}, t_{0}\right)=I_{6 \times 6}
$$

( $I_{6 \times 6}$ is the 6 by 6 identity matrix). Letting $\tau=t-t_{0}$, an analytical expression exists for $\Theta$,

$$
\left.\begin{array}{ccc}
\frac{s(\omega \tau)}{\omega} & \frac{2[1-c(\omega \tau)]}{\omega} & 0 \\
\frac{[c(\omega \tau)-1]}{\omega} & \frac{4 s(\omega \tau)-3 \omega \tau}{\omega} & 0 \\
0 & 0 & \frac{s(\omega \tau)}{\omega} \\
c(\omega \tau) & 2 s(\omega \tau) & 0 \\
-2 s(\omega \tau) & 4 c(\omega \tau)-3 & 0 \\
0 & 0 & c(\omega \tau)
\end{array}\right],
$$

$$
\begin{aligned}
& \sin \xi=\frac{z_{0}}{c_{4}}, \\
& \cos \xi=\frac{\dot{z}_{0}}{\omega c_{4}} .
\end{aligned}
$$

Equations (9)-(12) allow identifying some special solutions to the homogeneous EHCW equations of motion. The following solution is of special interest.

(a) Relative elliptic motion, if $c_{2}=0$ : this special solution corresponds to the absence (in (10)) of the second term, responsible for the drift of $P$ with respect to $T$. If also $c_{4}=0$ then the ellipse lies in the $\left(\widehat{r}_{T}, \widehat{\theta}_{T}\right)$-plane (the spacecraft motion is two-dimensional).

From inspecting (9)-(11) it is apparent that the dynamics of the (out-of-plane) components of position and velocity (i.e., $z$ and $\dot{z}$ ) are completely uncoupled from the dynamics of the remaining (in-plane) components. As an immediate consequence, the analysis and optimization of planar cases (involving only the components $x$ and $y$ ) can be performed by simply setting $z=0$ and $\dot{z}=0$ and neglecting the respective equations, which become unnecessary.

\section{Optimal Relative Orbit Trajectories}

In general, in relative orbit maneuvering two types of optimal paths are sought: (a) trajectories that minimize fuel expenditure in a given time and (b) paths that minimize the time of flight. They are related to specific analytical conditions for optimality, which are the subject of this section.

3.1. Optimal Thrust Programming for Minimum-Fuel Relative Trajectories. This subsection is focused on the problem of minimizing the fuel consumption needed for performing orbital rendezvous or transfers in a specified time $\Delta t\left(=t_{f}-\right.$ $t_{i}$ ). The thrust acceleration $\mathbf{a}(t)$ (with magnitude denoted by 
$\left.a_{P}(t)\right)$ represents the control authority over the dynamics of $P$. The pursuing spacecraft employs a time-varying thrust (with magnitude $T(t)$ ), and therefore $a_{P}(t)$ is given by $a_{P}(t)=$ $T(t) / m(t)$, with $T(t)$ constrained to $\left[0, T_{\max }\right]$. Moreover, letting $x_{7}:=m / m_{i}$ (where $m_{i}$ is the initial spacecraft mass), the following additional (scalar) state equation is introduced:

$$
\dot{x}_{7}=-\frac{n}{c}, \quad \text { where } n(t):=\frac{T(t)}{m_{i}} .
$$

The component $x_{7}$ is included in the state vector $\mathbf{x}$ (:= $\left[\begin{array}{ll}\widetilde{\mathbf{x}}^{T} & x_{7}\end{array}\right]^{T}$ ). In (13) $c$ is the effective exhaust velocity of the propulsive system, which is assumed constant. The thrust direction is defined by two angles: $\beta$ (out-of-plane angle), constrained to $[-\pi / 2, \pi / 2]$, and $\alpha$ (in-plane angle), constrained to $[-\pi, \pi]$. As a result, the thrust acceleration components are

$$
\begin{aligned}
& a_{x}=\left(\frac{n}{x_{7}}\right) \cos \beta \sin \alpha, \\
& a_{y}=\left(\frac{n}{x_{7}}\right) \cos \beta \cos \alpha, \\
& a_{z}=\left(\frac{n}{x_{7}}\right) \sin \beta .
\end{aligned}
$$

The formulation of the optimal control problem is thus the following: determine the optimal thrust magnitude and direction that minimize fuel consumption; that is,

$$
\begin{aligned}
& \mathbf{u}^{*}(t)=\arg \min _{\mathbf{u}} j, \\
& \text { where } \mathbf{u}^{*}=\left[\begin{array}{lll}
n & \alpha & \beta
\end{array}\right]^{T}, J=\int_{t_{i}}^{t_{f}} n(t) d t,
\end{aligned}
$$

where $\mathbf{u}$ represents the control vector. Constraints for the problem at hand are equations of motion (4) and (13) and boundary conditions of the form

$$
\psi\left(\mathbf{x}_{i}, \mathbf{x}_{f}, t_{f}\right)=\mathbf{0}
$$

where the subscripts " $i$ " and " $f$ " denote the initial and final values of the respective variable.

A Hamiltonian $H$ and a function of the terminal conditions $\Phi$ are introduced, with the intent of deriving the necessary conditions for optimality [34]

$$
\begin{aligned}
& H=\tilde{\lambda}^{T}(A \mathbf{x}+\mathbf{a})-\frac{\lambda_{7} n}{c}+n, \\
& \Phi=\boldsymbol{v}^{T} \psi
\end{aligned}
$$

where $\lambda:=\left[\begin{array}{ll}\tilde{\lambda}^{T} & \lambda_{7}\end{array}\right]^{T}$ and $\boldsymbol{v}$ are the adjoint variables conjugate to the dynamics equations and to the boundary conditions, respectively. First, the Pontryagin minimum principle [34] assumes the form

$$
\begin{aligned}
& {\left[\begin{array}{l}
n^{*} \\
\alpha^{*} \\
\beta^{*}
\end{array}\right]=\arg \min _{\{n, \alpha, \beta\}} H=\arg \min _{\{n, \alpha, \beta\}}\left[\frac{n}{x_{7}}\right.} \\
& \cdot\left(\lambda_{4} \cos \beta \sin \alpha+\lambda_{5} \cos \beta \cos \alpha+\lambda_{6} \sin \beta\right)+n \\
& \left.-\frac{\lambda_{7} n}{c}\right]
\end{aligned}
$$

(the symbol “*” denotes the optimal time history of the respective control variable). As the term $\left(n / x_{7}\right)$ is nonnegative, for $\left\{\alpha^{*}, \beta^{*}\right\}$ (18) can be rewritten as

$$
\begin{aligned}
& {\left[\begin{array}{l}
\alpha^{*} \\
\beta^{*}
\end{array}\right]} \\
& =\arg \min _{\{\alpha, \beta\}} H \\
& \left.=\arg \min _{\{\alpha, \beta\}}\left\{\begin{array}{lll}
\lambda_{4} & \lambda_{5} & \lambda_{6}
\end{array}\right]\left[\begin{array}{lll}
\cos \beta \sin \alpha & \cos \beta \cos \alpha & \sin \beta
\end{array}\right]^{T}\right\}
\end{aligned}
$$

and yields the following relations:

$$
\begin{aligned}
& \sin \alpha^{*}=-\frac{\lambda_{4}}{\sqrt{\lambda_{4}^{2}+\lambda_{5}^{2}}}, \\
& \cos \alpha^{*}=-\frac{\lambda_{5}}{\sqrt{\lambda_{4}^{2}+\lambda_{5}^{2}}}, \\
& \sin \beta^{*}=-\frac{\lambda_{6}}{\sqrt{\lambda_{4}^{2}+\lambda_{5}^{2}+\lambda_{6}^{2}}} .
\end{aligned}
$$

Equations (20) are meaningless during coast intervals. After inserting (20), (18) becomes

$$
\begin{aligned}
n^{*} & =\arg \min _{n} H \\
& =\arg \min _{n}\left[n\left(1-\frac{\lambda_{7}}{c}-\frac{\sqrt{\lambda_{4}^{2}+\lambda_{5}^{2}+\lambda_{6}^{2}}}{x_{7}}\right)\right] .
\end{aligned}
$$

The term in parentheses is usually referred to as the switching function $S$, because it determines the optimal sequence of thrust and coast intervals. In fact, under the assumption that $S$ does not vanish over finite time intervals (i.e., excluding singular-control arcs), (21) yields the optimal thrust magnitude

$$
n^{*}=\left\{\begin{array}{ll}
0 & \text { if } S>0 \\
n_{\max } & \text { if } S<0
\end{array} \quad\left(n_{\max }:=\frac{T_{\max }}{m_{i}}\right) .\right.
$$

This means that minimum-fuel trajectories are composed of maximum-thrust intervals and coast arcs. Their optimal 
sequence is established by $S$, according to (22). Moreover, the adjoint (or costate) equations for $\lambda$ are [34]

$$
\begin{gathered}
\dot{\tilde{\lambda}}=-\left[\frac{\partial H}{\partial \widetilde{\mathbf{x}}}\right]^{T}=-A^{T} \widetilde{\lambda}, \\
\dot{\lambda}_{7}=-\frac{n}{x_{7}^{2}} \sqrt{\lambda_{4}^{2}+\lambda_{5}^{2}+\lambda_{6}^{2}} .
\end{gathered}
$$

$$
\widetilde{\Theta}(\tau)=\left[\begin{array}{cccccc}
4-3 c(\omega \tau) & 6 \omega t-6 s(\omega \tau) & 0 & -3 \omega s(\omega \tau) & -6 \omega[1-c(\omega \tau)] & 0 \\
0 & 1 & 0 & 0 & 0 & 0 \\
0 & 0 & \cos (\omega \tau) & 0 & 0 & \omega s(\omega \tau) \\
-\frac{s(\omega \tau)}{\omega} & -2 \frac{[1-c(\omega \tau)]}{\omega} & 0 & c(\omega \tau) & 2 s(\omega \tau) & 0 \\
\frac{2[1-c(\omega \tau)]}{\omega} & \frac{3 \omega \tau-4 s(\omega \tau)}{\omega} & 0 & -2 s(\omega \tau) & 4 c(\omega \tau)-3 & 0 \\
0 & 0 & -\frac{s(\omega \tau)}{\omega} & 0 & 0 & c(\omega \tau)
\end{array}\right] .
$$

It is worth remarking that the adjoint vector $\tilde{\lambda}$ has the same analytical form along thrust intervals and coast arcs. The remaining necessary conditions regard the initial and final times, $t_{i}$ and $t_{f}$. Since these conditions are problemdependent, only their general form is reported,

$$
\begin{aligned}
& \lambda_{i}=-\left[\frac{\partial \Phi}{\partial \mathbf{x}_{i}}\right]^{T} \\
& \lambda_{f}=\left[\frac{\partial \Phi}{\partial \mathbf{x}_{f}}\right]^{T}
\end{aligned}
$$

where the subscripts " $i$ " and " $f$ " refer, respectively, to $t_{i}$ and $t_{f}$. As $x_{7 f}$ is unspecified, $\lambda_{7 f}=0$ for all of the cases that are being considered.

\subsection{Optimal Thrust Programming for Minimum-Time Relative} Trajectories. In this subsection the problem of determining minimum-time rendezvous and transfer trajectories in the Euler-Hill frame is considered. The thrust acceleration $\mathbf{a}(t)$ represents again the control authority over the dynamics of $P$ and has components given by (5). The state vector includes the same variables introduced in the preceding subsection; the initial time $t_{i}$ is specified (and set to 0 ), whereas the final time $t_{f}$ is unspecified and is to be minimized.

The formulation of the optimal control problem is thus the following: determine the optimal thrust magnitude and direction that minimize the time of flight; that is,

$$
\begin{aligned}
& \mathbf{u}^{*}(t)=\arg \min _{\mathbf{u}} J \\
& \qquad \text { where } \mathbf{u}^{*}=\left[\begin{array}{lll}
n & \alpha & \beta
\end{array}\right]^{T}, J=\int_{0}^{t_{f}} d t,
\end{aligned}
$$

The six components of $\tilde{\lambda}$ can be expressed in closed form, after deriving the state transition matrix $\widetilde{\Theta}$ for the linear system (23) (first equation),

$$
\tilde{\lambda}(t)=\widetilde{\Theta}\left(t, t_{0}\right) \tilde{\lambda}\left(t_{0}\right) .
$$

The matrix $\widetilde{\Theta}$ fulfills a differential equation formally identical to (7), with $-A^{T}$ in place of $A$. Letting $\tau=t-t_{0}, \widetilde{\Theta}$ assumes the following form: where $\mathbf{u}$ represents the control vector. Constraints for the problem at hand are equations of motion (4) and (13) and boundary conditions of form (16).

A Hamiltonian $H$ and a function of the terminal conditions $\Phi$ are introduced, with the purpose of deriving the necessary conditions for optimality,

$$
\begin{aligned}
& H=\tilde{\lambda}^{T}(A \mathbf{x}+\mathbf{a})+\lambda_{7}\left(-\frac{n}{c}\right)+1, \\
& \Phi=\boldsymbol{v}^{T} \psi .
\end{aligned}
$$

The Pontryagin minimum principle assumes the form

$$
\begin{aligned}
& {\left[\begin{array}{l}
n^{*} \\
\alpha^{*} \\
\beta^{*}
\end{array}\right]=\arg \min _{(n, \alpha, \beta)} H=\arg \min _{(n, \alpha, \beta)}\left[\frac{n}{x_{7}}\right.} \\
& \cdot\left(\lambda_{4} \cos \beta \sin \alpha+\lambda_{5} \cos \beta \cos \alpha+\lambda_{6} \sin \beta\right) \\
& \left.-\frac{\lambda_{7} n}{c}\right] .
\end{aligned}
$$

As the term $\left(n / x_{7}\right)$ is nonnegative, (29) yields again relations (20). After inserting (20), (29) becomes

$$
\begin{aligned}
n^{*} & =\arg \min _{n} H \\
& =\arg \min _{n}\left[n\left(-\frac{\lambda_{7}}{c}-\frac{\sqrt{\lambda_{4}^{2}+\lambda_{5}^{2}+\lambda_{6}^{2}}}{x_{7}}\right)\right] .
\end{aligned}
$$

Due to (28), the adjoint equations are the same as those reported in (23), with related boundary conditions (26), which yield again $\lambda_{7 f}=0$, because $x_{7 f}$ is unspecified. 
The latter condition, in conjunction with the adjoint equation for $\lambda_{7}$, implies that $\lambda_{7}>0\left(0 \leq t<t_{f}\right)$. As a result, the term in parentheses in (30) is always negative, and this leads to determining the optimal thrust magnitude that minimizes the Hamiltonian; that is,

$$
n(t)=n_{\max }, \quad 0 \leq t \leq t_{f}
$$

Moreover, as $t_{f}$ is unspecified, the transversality condition holds,

$$
H_{f}+\frac{\partial \Phi}{\partial t_{f}}=0 \Longrightarrow H_{f}=0
$$

\section{Symmetry Properties}

This section addresses a pair of symmetry properties that hold in the presence of certain boundary conditions:

(a) symmetry of two-dimensional and three-dimensional minimum-fuel and minimum-time paths,

(b) symmetry of the optimal thrust direction and sequence for two-dimensional minimum-fuel trajectories.

4.1. First Symmetry Property. The first interesting symmetry property regards minimum-fuel and minimum-time trajectories in the Euler-Hill frame. In both cases, the adjoint variables have the same (analytical) expressions and determine the optimal thrust direction (cf. (20)), as well as the optimal thrust sequence of minimum-fuel paths (cf. (22)).

Proposition 1. Let $\mathbf{X}_{A}^{*}:=\left\{\widetilde{\mathbf{x}}_{A}^{*}, x_{7 A}^{*}, n_{A}^{*}, \alpha_{A}^{*}, \beta_{A}^{*}\right\}$ be the optimal solution of problem $A$, which is in determining either the minimum-fuel (I) or the minimum-time (II) relative trajectory,

$$
\begin{gathered}
\text { (I) } J=\int_{t_{i}}^{t_{f}} n d t \quad\left(t_{i} \text { and } t_{f} \text { specified }\right) \\
(I I) J=\int_{t_{i}}^{t_{f}} d t \quad\left(t_{i}=0, t_{f} \text { unspecified }\right),
\end{gathered}
$$

while holding equations of motion (4) and (13) and the boundary conditions

$$
\psi^{(A)}\left(\widetilde{\mathbf{x}}_{i}, \widetilde{\mathbf{x}}_{f}, t_{f}\right)=\mathbf{0} .
$$

The set $\mathbf{X}_{B}:=\left\{\widetilde{\mathbf{x}}_{B}=-\widetilde{\mathbf{x}}_{A}^{*}, x_{7 B}=x_{7 A}^{*}, n_{B}=n_{A}^{*}, \alpha_{B}=\pi+\right.$ $\left.\alpha_{A}^{*}, \beta_{B}=-\beta_{A}^{*}\right\}$ is the optimal solution to problem $B$, formulated either as (I) or as (II), with boundary conditions

$$
\psi^{(B)}\left(\widetilde{\mathbf{x}}_{i}, \widetilde{\mathbf{x}}_{f}, t_{f}\right)=\mathbf{0}
$$

provided that

$$
\psi^{(B)}\left(\widetilde{\mathbf{x}}_{i}, \widetilde{\mathbf{x}}_{f}, t_{f}\right) \equiv \psi^{(A)}\left(-\widetilde{\mathbf{x}}_{i},-\widetilde{\mathbf{x}}_{f}, t_{f}\right)
$$

Proof. By definition, the solution $X_{A}^{*}$ meets the necessary conditions for optimality, that is, (20) and (23)-(26), in conjunction with (22) for objective (I) and (31) and (32) for objective (II). Omitting the superscript “*” for the elements of solution $A$ henceforward, (26) yield

$$
\begin{aligned}
\tilde{\lambda}_{A i} & =-\left[\frac{\partial \psi^{(A)}}{\partial \widetilde{\mathbf{x}}_{i}}\right]^{T} \boldsymbol{v}_{A}, \\
\tilde{\lambda}_{A f} & =\left[\frac{\partial \psi^{(A)}}{\partial \widetilde{\mathbf{x}}_{f}}\right]^{T} \boldsymbol{v}_{A}, \\
\lambda_{7 A f} & =0 .
\end{aligned}
$$

Now, if for problem $B$ the adjoint vectors $\lambda_{7 B}, \tilde{\lambda}_{B}$, and $\boldsymbol{v}_{B}$ are assumed to be given by

$$
\begin{aligned}
\tilde{\lambda}_{B} & =-\tilde{\boldsymbol{\lambda}}_{A}, \\
\lambda_{7 B} & =\lambda_{7 A}, \\
\boldsymbol{v}_{B} & =\boldsymbol{v}_{A}
\end{aligned}
$$

then adjoint equations (23) and related boundary conditions (26) are fulfilled by $\lambda_{7 B}, \tilde{\lambda}_{B}$, and $v_{B}$. In fact, the adjoint equations, written for $\tilde{\lambda}_{B}$, reduce to those holding for $\tilde{\lambda}_{A}$. In addition, due to (36), the boundary conditions for $\tilde{\lambda}_{A}$ imply satisfaction of the corresponding boundary conditions for $\widetilde{\lambda}_{B}$,

$$
\begin{aligned}
& \tilde{\lambda}_{A i}=-\left[\frac{\partial \psi^{(A)}}{\partial \widetilde{\mathbf{x}}_{i}}\right]^{T} \boldsymbol{v}_{A} \Longleftrightarrow \tilde{\lambda}_{B i}=-\left[\frac{\partial \psi^{(B)}}{\partial \mathbf{x}_{i}}\right]^{T} \boldsymbol{v}_{B}, \\
& \tilde{\lambda}_{A f}=\left[\frac{\partial \psi^{(A)}}{\partial \widetilde{\mathbf{x}}_{f}}\right]^{T} \boldsymbol{v}_{A} \Longleftrightarrow \tilde{\lambda}_{B f}=\left[\frac{\partial \psi^{(B)}}{\partial \mathbf{x}_{f}}\right]^{T} \boldsymbol{v}_{B} .
\end{aligned}
$$

With regard to the control variables, (22) (for objective (I)) and (31) (for objective (II)) in conjunction with (20) and (38) yield

$$
\begin{gathered}
n_{B}=n_{A}^{*}, \\
\sin \alpha_{B}=-\sin \alpha_{A}^{*}, \\
\cos \alpha_{B}=-\cos \alpha_{A}^{*}, \\
\sin \beta_{B}=-\sin \beta_{A}^{*}, \\
\Longleftrightarrow \alpha_{B}=\pi+\alpha_{A}^{*}, \quad \beta_{B}=-\beta_{A}^{*} .
\end{gathered}
$$

Due to the previous relations on $\tilde{\lambda}_{B}$ and $n_{B}$, if $\lambda_{7 B}=\lambda_{7 A}$, then the adjoint equation for $\lambda_{7}$ and the related boundary condition $\left(\lambda_{7 B f}=0\right)$ are both satisfied. Insertion of the control variables $\left\{n_{B}, \alpha_{B}, \beta_{B}\right\}$ in the state equations (1) leads to their fulfillment, because these equations reduce to the corresponding equations that hold for solution $A$. In conclusion, since all the necessary conditions for optimality are met, $X_{B}$ is the optimal solution for problem $B$, formulated either as minimum-fuel (I) or as minimum-time (II) problem. 


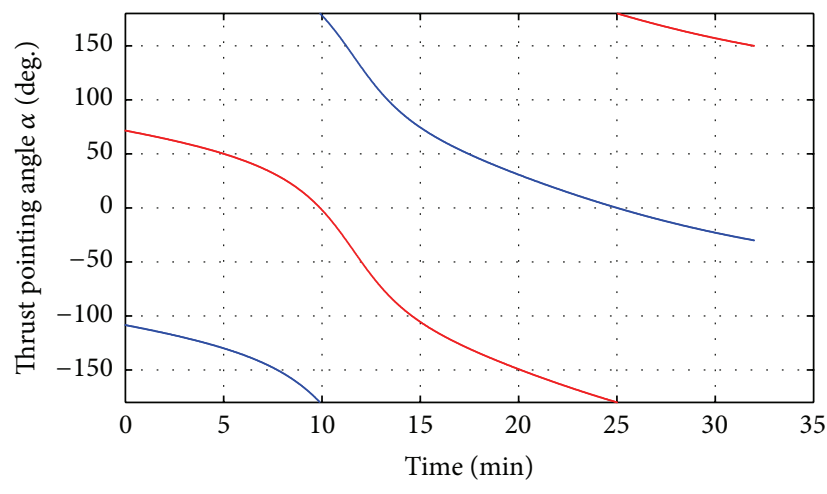

(a)

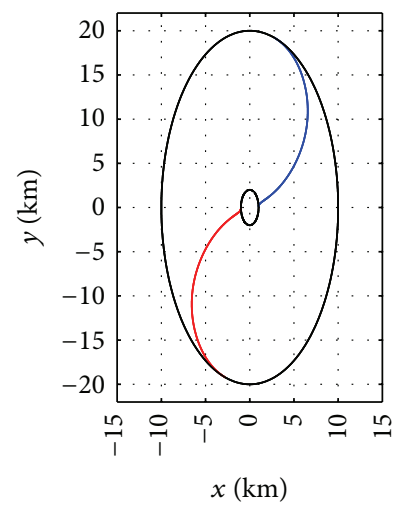

(b)

FIGURE 1: Relative orbit transfer: optimal control time histories (a) and optimal trajectories (b).
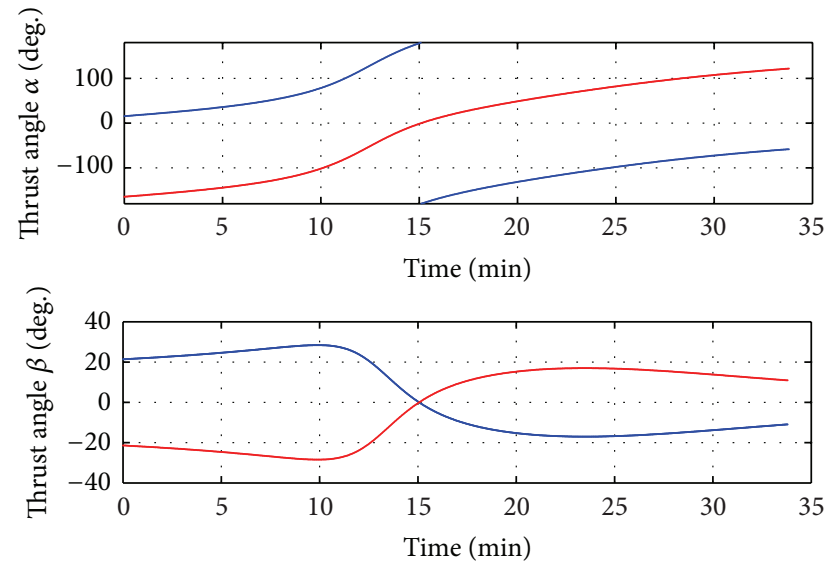

(a)

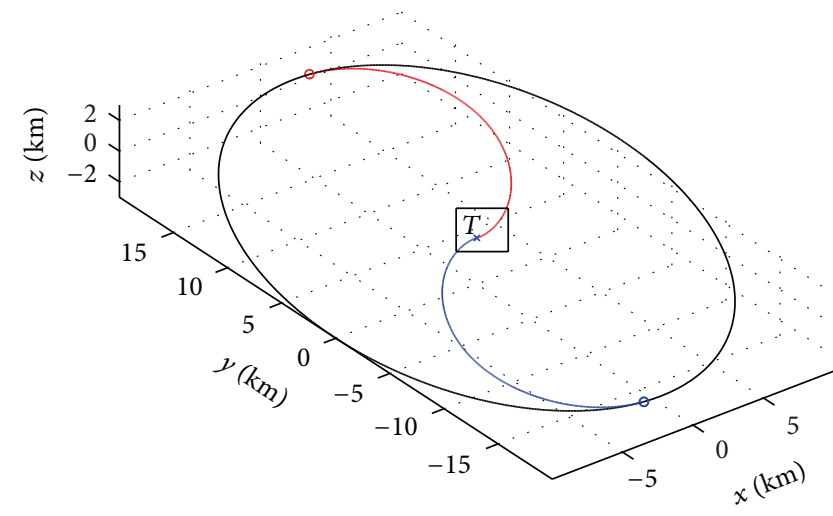

(b)

FIgURE 2: Orbital rendezvous: optimal control time histories (a) and optimal trajectories (b).

4.2. Examples of Symmetric Minimum-Time Relative Trajectories. Two examples that fulfill the conditions stated in Proposition 1 are reported in this subsection, for the purpose of illustrating the previously described symmetry property. The reference circular orbit around the Earth has an altitude of $400 \mathrm{~km}$. The thrust magnitude is set to $0.01 \mathrm{~m} / \mathrm{sec}^{2}$, whereas the effective exhaust velocity is $25 \mathrm{~km} / \mathrm{sec}$.

(a) Transfer between two coplanar relative elliptic orbits (Figure 1): the problem consists in determining (i) the departure point from the periodic orbit (with semimajor axis of $20 \mathrm{~km}$ ) and (ii) the thrust direction that minimize the time needed to inject the pursuing spacecraft in a different coplanar relative elliptic orbit (with semimajor axis of $2 \mathrm{~km}$ ).

(b) Three-dimensional rendezvous from a relative elliptic orbit (Figure 2): the problem consists in determining (i) the departure point from the periodic orbit and (ii) the thrust direction that minimizes the time needed to rendezvous with the target, denoted by $T$ in Figure 2(b). The initial relative elliptic orbit is associated with the position coordinates (4)-(6), with $c_{2}=c_{3}=0, c_{1}=10 \mathrm{~km}, c_{4}=3 \mathrm{~km}$, and $\xi=\zeta$. The condition $c_{2}=0$ corresponds to relative elliptic orbits (as previously remarked), $c_{3}=0$ implies symmetry of the relative orbit with respect to the target location, whereas the values $c_{1}$ and $c_{4}$ and the angular relation define, respectively, the ellipse size and spatial orientation.

Further details pertaining to computational aspects are reported in [32]. It is apparent that the utility of Proposition 1 is in determining immediately the second optimal path and the related optimal control time history, once the first optimal trajectory has been found. This may be relevant in the presence of specific practical constraints (e.g., if the transfer must be performed at the earliest occurrence, one can choose between the two options represented by trajectory 1 and path 2 , with no difference in terms of time of flight and propellant expenditure).

4.3. Second Symmetry Property. A further symmetry property concerns minimum-fuel two-dimensional trajectories (i.e., $z=0, v_{z}=0, \lambda_{6}=0$, and $\beta=0 \forall t$ ). As a preliminary 


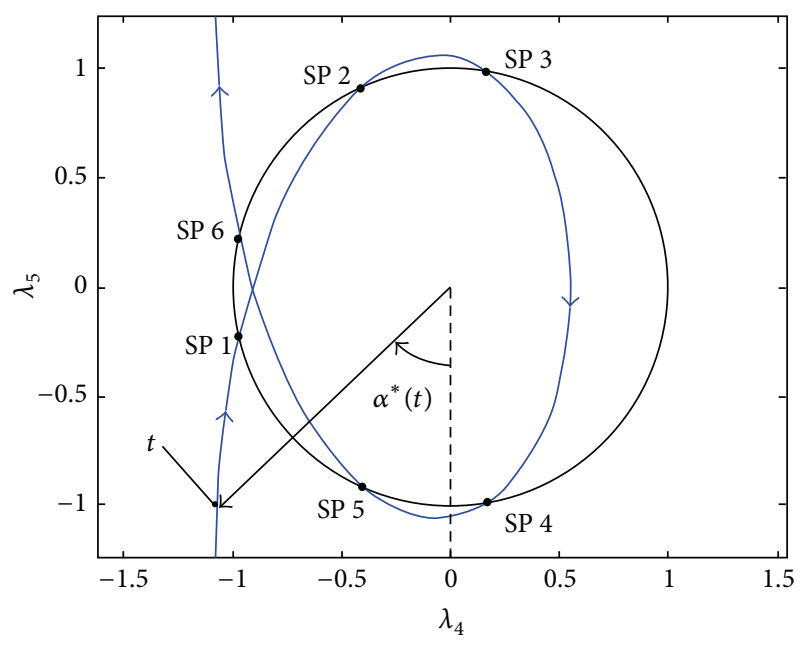

Figure 3: Cycloid associated with $\lambda_{4}$ and $\lambda_{5}$ (SP denotes switching points).

step, from inspection of (20) and (22) it is apparent that the thrust direction and magnitude depend only on the adjoint variables $\left(\lambda_{4}, \lambda_{5}\right)$. It is relatively straightforward to rewrite their analytical expressions as

$$
\begin{aligned}
& \lambda_{4}=C \cos (\omega \tau+\varphi)-\frac{2 \lambda_{20}}{\omega}, \\
& \lambda_{5}=-2 C \sin (\omega \tau+\varphi)+D+3 \lambda_{20} \tau,
\end{aligned}
$$

where $C, D$, and $\varphi$ depend on $\lambda_{10}, \lambda_{20}, \lambda_{30}$, and $\lambda_{40}$ (the subscript " 0 " corresponds to $\tau=0$ ). Under the assumption that $C \neq 0$, the geometrical locus associated with $\left(\lambda_{4}, \lambda_{5}\right)$ is either an ellipse (if $\lambda_{20}=0$ ) or a cycloid (if $\lambda_{20} \neq 0$ ). Figure 3 illustrates a cycloid in an orbital period and shows the optimal control direction, determined using (20).

If the effective exhaust velocity is very high, then the mass depletion rate is modest, and the following approximation can be adopted: $c \rightarrow \infty$. This implies that $x_{7}=1 \forall t$. As a result, (13) becomes unnecessary, as well as the respective adjoint equation for $\lambda_{7}$ (cf. (23)). Under this assumption the switching function assumes the form

$$
S=1-\sqrt{\lambda_{4}^{2}+\lambda_{5}^{2}}
$$

Hence, in the $\left(\lambda_{4}, \lambda_{5}\right)$-plane the condition for thrust phases to occur $(S<0)$ corresponds to the cycloid (or ellipse) arcs that lie outside the unit circle. Intersections of the cycloid with the unit circle correspond to the switching times between thrust intervals and coast arcs. In an orbital period, their maximum number equals 6 (for a cycloid) or 4 (for an ellipse). This means that at most four powered phases, separated by three coast arcs, can occur. This property has been well known for impulsive relative paths since the 1960s $[7,8]$ and holds also for finite-thrust trajectories, due to the analytic form of the adjoint variables $\lambda_{4}$ and $\lambda_{5}$, as also remarked by Carter [24].
Proposition 2. With regard to two-dimensional minimumfuel paths, if the maximal thrust acceleration is constant, that is, $n_{\max } / x_{7} \equiv n_{\max } \forall t$, and if

$$
\begin{aligned}
& x_{1 f}=x_{1 i}, \\
& x_{5 f}=x_{5 i}
\end{aligned}
$$

then (i) the optimal thrust sequence is symmetric with respect to the intermediate time $t_{M}$ and (ii) the optimal thrust angle $\alpha^{*}$ fulfills the following relation:

$$
\begin{aligned}
& \alpha^{*}\left(t-t_{M}\right) \\
& \quad= \begin{cases}-\pi-\alpha^{*}\left(t_{M}-t\right) & \text { if }-\pi<\alpha^{*}\left(t_{M}-t\right) \leq 0 \\
\pi-\alpha^{*}\left(t_{M}-t\right) & \text { if } 0 \leq \alpha^{*}\left(t_{M}-t\right) \leq \pi .\end{cases}
\end{aligned}
$$

Proof. As a first step, the time variable $\tau$ is defined as $\tau:=$ $t-t_{M}\left(t_{M}:=t_{i}+\Delta t / 2\right)$. Then, the state components $x_{1}(\tau)$ and $x_{5}(\tau)$ are easily obtained from (6):

$$
\begin{aligned}
& x_{1}(\tau)=[4-3 \cos (\omega \tau)] x_{10}+\frac{\sin (\omega \tau)}{\omega} x_{40}+\frac{2}{\omega}[1 \\
& -\cos (\omega \tau)] x_{50}+\int_{0}^{\tau}\left\{n \left[\frac{\sin (\omega \xi)}{\omega} \sin (\alpha(\xi))\right.\right. \\
& \left.\left.+\frac{2}{\omega}[1-\cos (\omega \xi)] \cos (\alpha(\xi))\right]\right\} d \xi, \\
& x_{5}(\tau)=-6 \omega[1-\cos (\omega \tau)] x_{10}-2 \sin (\omega \tau) x_{40}+[4 \\
& \quad \cdot \cos (\omega \tau)-3] x_{50}+\int_{0}^{\tau}\{n[-2 \sin (\omega \xi) \sin (\alpha(\xi)) \\
& \quad+[4 \cos (\omega \xi)-3] \cos (\alpha(\xi))]\} d \xi .
\end{aligned}
$$

It is worth noticing that in this subsection the subscript " 0 " corresponds to the intermediate time $t_{M}$ (i.e., $\tau=0$ ). Equations (45) are evaluated at the initial time $(\tau=-\Delta t / 2)$ and at the final time $(\tau=\Delta t / 2)$; then, they are inserted in (43), to yield

$$
\begin{aligned}
& 2 x_{40} \frac{\sin (\omega \Delta t / 2)}{\omega}+\int_{-\Delta t / 2}^{\Delta t / 2}\left\{n \left[\frac{\sin (\omega \xi)}{\omega} \sin (\alpha(\xi))\right.\right. \\
& \left.\left.+\frac{2}{\omega}[1-\cos (\omega \xi)] \cos (\alpha(\xi))\right]\right\} d \xi=0, \\
& -4 x_{40} \sin (\omega \Delta t / 2)+\int_{-\Delta t / 2}^{\Delta t / 2}\{n[-2 \sin (\omega \xi) \sin (\alpha(\xi)) \\
& +[4 \cos (\omega \xi)-3] \cos (\alpha(\xi))]\} d \xi=0 .
\end{aligned}
$$

After combining (46), the following relation is obtained:

$$
\int_{-\Delta t / 2}^{\Delta t / 2} n \cos [\alpha(\xi)] d \xi=0 .
$$

Due to the geometrical properties of the $\left(\lambda_{4}, \lambda_{5}\right)$-locus, (47) is satisfied only if in the interval $t_{i} \leq t \leq t_{f}$ (i.e., $-\Delta t / 2 \leq$ 
$\tau \leq \Delta t / 2$ ) the portion of cycloid (or ellipse, in the degenerate case $\lambda_{20}=0$ ) is symmetrical with respect to the $\lambda_{4}$-axis. This implies also that the following two properties hold: (a) the function $\cos [\alpha(\tau)]$ is odd and (b) the function $\sin [\alpha(\tau)]$ is even. Properties (a) and (b) are equivalent to (44) (statement (ii)). In addition, symmetry of the $\left(\lambda_{4}, \lambda_{5}\right)$-locus implies also the symmetry of the optimal thrust sequence (statement (i)).

Due to the analytical form of $\widetilde{\Theta}$ (appearing in (25)), it is also immediate to recognize that (a) $\lambda_{4}(\tau)$ is even and (b) $\lambda_{5}(\tau)$ is odd. As a result,

$$
\begin{aligned}
& \lambda_{4 i}=\lambda_{4 f}, \\
& \lambda_{50}=0 .
\end{aligned}
$$

As an immediate consequence of the previously mentioned symmetry property, the following integral equalities can be easily derived, by considering the properties of odd and even functions of time:

$$
\begin{aligned}
& \int_{0}^{\Delta t / 2} n \xi \cos [\alpha(\xi)] d \xi-\int_{-\Delta t / 2}^{0} n \xi \cos [\alpha(\xi)] d \xi=0 \\
& \int_{0}^{\Delta t / 2} n \cos (\omega \xi) \sin [\alpha(\xi)] d \xi \\
& -\int_{-\Delta t / 2}^{0} n \cos (\omega \xi) \sin [\alpha(\xi)] d \xi=0 \\
& \int_{0}^{\Delta t / 2} n \sin (\omega \xi) \cos [\alpha(\xi)] d \xi \\
& -\int_{-\Delta t / 2}^{0} n \sin (\omega \xi) \cos [\alpha(\xi)] d \xi=0 .
\end{aligned}
$$

These relations can be profitably employed to obtain the values of $x_{2}$ and $x_{4}$ at $t_{M}$, that is, $x_{20}$ and $x_{40}$. With regard to $x_{4}$, the use of the related analytic expression deriving from (6) (evaluated at $\tau=-\Delta t / 2$ and $\tau=\Delta t / 2$ ), together with (50) and (51), yields

$$
\begin{aligned}
& x_{4 i}+x_{4 f}=2 x_{40} \cos (\omega \Delta t / 2) \\
& \Longrightarrow x_{40}=\frac{x_{4 i}+x_{4 f}}{2 \cos (\omega \Delta t / 2)} .
\end{aligned}
$$

Then, the corresponding expression for $x_{2}$, together with (49)-(51), leads to the following relationship:

$$
\begin{aligned}
x_{2 i}+x_{2 f} & =2 x_{20}-\frac{4 x_{40}}{\omega}\left[1-\cos \left(\frac{\omega \Delta t}{2}\right)\right] \\
\Longrightarrow x_{20} & =\frac{x_{2 i}+x_{2 f}}{2}+\frac{2 x_{40}}{\omega}\left[1-\cos \left(\frac{\omega \Delta t}{2}\right)\right] .
\end{aligned}
$$

Equations (52) and (53) prove that the values of $x_{2}$ and $x_{4}$ at the intermediate time depend only on the respective initial and final values, as well as on the time of flight $\Delta t$.

Lastly, due to the analytic form of the adjoint variables, (48) yield useful relations among the initial values of the costate variables. In fact, letting $t_{i}=0, \lambda_{4}$ and $\lambda_{5}$ can be rewritten as

$$
\begin{aligned}
\lambda_{4}(t)= & -\frac{\sin (\omega t)}{\omega} \lambda_{1 i}-\frac{2}{\omega}[1-\cos (\omega t)] \lambda_{2 i} \\
& +\cos (\omega t) \lambda_{4 i}+2 \sin (\omega t) \lambda_{5 i} \\
\lambda_{5}(t)= & \frac{2}{\omega}[1-\cos (\omega t)] \lambda_{1 i}+\frac{3 \omega t-4 \sin (\omega t)}{\omega} \lambda_{2 i} \\
& -2 \sin (\omega t) \lambda_{4 i}+[4 \cos (\omega t)-3] \lambda_{5 i} .
\end{aligned}
$$

Letting $c()=\cos ()$ and $s()=\sin ()$, after inserting these expressions in (48), one obtains

$$
\begin{aligned}
& -\frac{s\left(\omega t_{f}\right)}{\omega} \lambda_{1 i}-\frac{2}{\omega}\left[1-c\left(\omega t_{f}\right)\right] \lambda_{2 i}+c\left(\omega t_{f}\right) \lambda_{4 i} \\
& \quad+2 s\left(\omega t_{f}\right) \lambda_{5 i}=\lambda_{4 i}, \\
& \frac{2}{\omega}\left[1-c\left(\frac{\omega t_{f}}{2}\right)\right] \lambda_{1 i}+\frac{3 \omega t_{f} / 2-4 s\left(\omega t_{f} / 2\right)}{\omega} \lambda_{2 i} \\
& \quad-2 s\left(\frac{\omega t_{f}}{2}\right) \lambda_{4 i}+\left[4 c\left(\frac{\omega t_{f}}{2}\right)-3\right] \lambda_{5 i}=0 .
\end{aligned}
$$

Equations (55) can be employed to derive two initial costate variables (e.g., $\lambda_{1 i}$ and $\lambda_{2 i}$ ) as functions of the remaining ones.

In conclusion, if the state components are specified at both the final and the initial time and boundary conditions (43) hold, then the numerical solution of the relative trajectory optimization problem may be sought

(a) in the time interval $[0, \Delta t / 2]$, instead of $[0, \Delta t]$,

(b) by using two initial values of the adjoint variables $\left\{\lambda_{4 i}, \lambda_{5 i}\right\}$ as unknown parameters, instead of four unknown parameters $\left\{\lambda_{1 i}, \lambda_{2 i}, \lambda_{4 i}, \lambda_{5 i}\right\}$,

(c) by assuming (52) and (53) as the only boundary conditions, without the need for enforcing the conditions on $x_{1}$ and $x_{5}$.

Points (b) and (c) imply that the parametric problem is reduced to half of its original size, whereas point (a) means that each trajectory is to be computed up to the intermediate time during the numerical solution process. These circumstances have very positive effects on computational efficiency. In fact, if the indirect heuristic method is used, halving the number of individuals and the propagation time (for each trajectory) implies reducing the computational runtime by a factor 4 .

4.4. Example of Symmetric Minimum-Fuel Relative Trajectory. The optimal relative trajectory presented in this subsection is very similar to the four-thrust-arc transfer treated by Carter [24]. The reference orbit is a low Earth circular orbit at an 


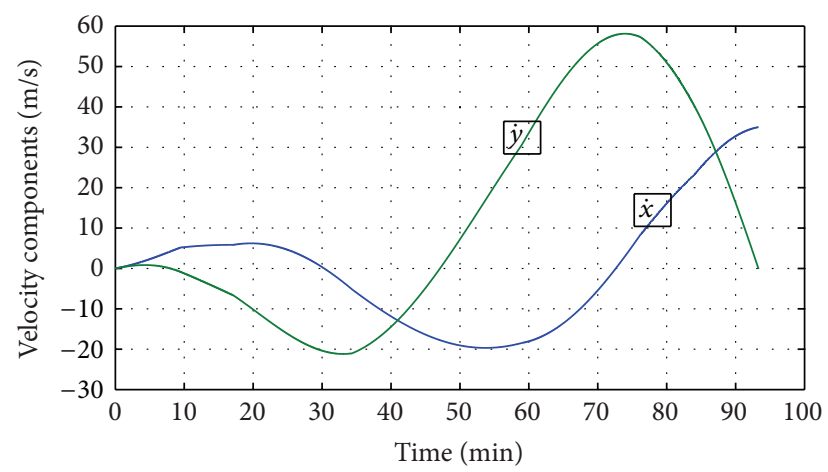

(a)

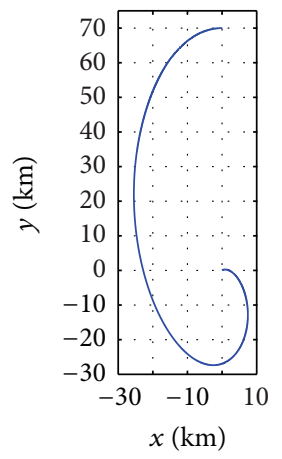

(b)

FIGURE 4: Minimum-fuel relative trajectory: velocity components (a) and optimal path (b).

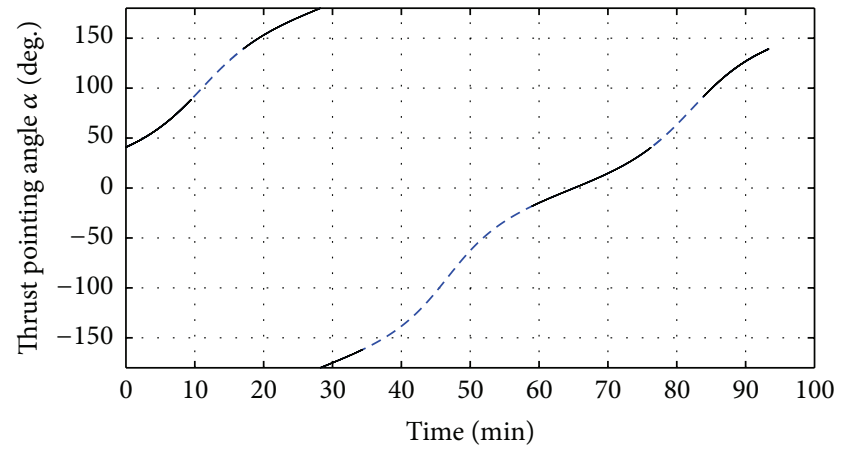

(a)

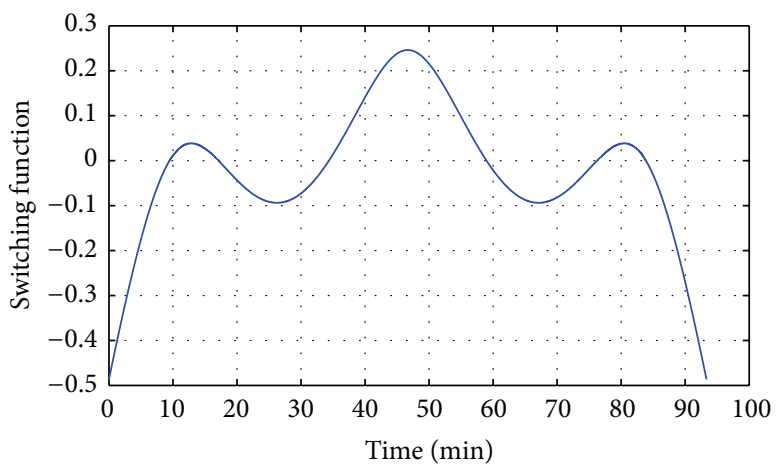

(b)

FIGURE 5: Minimum-fuel relative trajectory: optimal thrust direction (a) and switching function (b).

altitude of $435 \mathrm{~km}$, while $n_{\max }=7.583 \cdot 10^{-3} \mathrm{~m} / \mathrm{sec}^{2}$ and $\Delta t=$ $93.33 \mathrm{~min}$. The boundary conditions are

$$
\begin{aligned}
x_{i} & =0 \mathrm{~km}, \\
y_{i} & =0 \mathrm{~km}, \\
z_{i} & =0 \mathrm{~km}, \\
\dot{x}_{i} & =0 \mathrm{~m} / \mathrm{sec}, \\
\dot{y}_{i} & =0 \mathrm{~m} / \mathrm{sec}, \\
\dot{z}_{i} & =0 \mathrm{~m} / \mathrm{sec}, \\
x_{f} & =0 \mathrm{~km}, \\
y_{f} & =70 \mathrm{~km}, \\
z_{f} & =0 \mathrm{~km}, \\
\dot{x}_{f} & =35 \mathrm{~m} / \mathrm{sec}, \\
\dot{y}_{f} & =0 \mathrm{~m} / \mathrm{sec}, \\
\dot{z}_{f} & =0 \mathrm{~m} / \mathrm{sec} .
\end{aligned}
$$

For this case special symmetry conditions (43) and (44) are satisfied and the optimal trajectory is composed of four powered phases. Figures 4 and 5 illustrate the optimal trajectory and the related velocity components, thrust pointing angle, and switching function. Further details pertaining to computational aspects are reported in [33].

\section{Conclusion}

This work is focused on a pair of symmetry properties that hold for optimal relative orbit trajectories. The existence of symmetry properties is proven in the presence of special boundary conditions for the problems of interest. First, both minimum-time and minimum-fuel paths are proven to enjoy a property that leads to defining the optimal trajectory (and the respective optimal control law) that is symmetrical with respect to a given, already determined optimal path. Minimum-fuel relative trajectories are composed of multiple powered phases (at most 4 in an orbital period), divided by coast arcs. The optimal thrust sequence, duration, and direction depend on the time evolution of the switching function and satisfy a second interesting symmetry property, provided that a pair of elementary boundary conditions holds. This allows halving the number of boundary conditions and unknown parameters with respect to the original formulation of the problem. Some examples illustrate the properties at hand, pointing out their doubtless interest and 
usefulness for the purpose of simplifying the determination of optimal relative orbit maneuvers.

\section{Conflict of Interests}

The author declares that there is no conflict of interests regarding the publication of this paper.

\section{References}

[1] D. F. Lawden, Optimal Trajectories for Space Navigation, Butterworths, London, UK, 1963.

[2] G. Leitmann, Variational Problems with Bounded Control Variables. Optimization Techniques, Academic Press, New York, NY, USA, 1962.

[3] A. Miele, "General variational theory of the flight paths of rocket-powered aircraft, missiles and satellite carriers," Astronautica Acta, vol. 4, pp. 11-21, 1958.

[4] T. N. Edelbaum, “Optimal space trajectories," Report 69-4, Analytical Mechanics Associates, 1969.

[5] J. P. Marec, Optimal Space Trajectories, Elsevier, New York, NY, USA, 1979.

[6] N. X. Vinh, "General theory of optimal trajectory for rocket flight in a resisting medium," Journal of Optimization Theory and Applications, vol. 11, no. 2, pp. 189-202, 1973.

[7] J. E. Prussing, "Optimal four-impulse fixed-time rendezvous in the vicinity of a circular orbit," AIAA Journal, vol. 7, pp. 928-935, 1969.

[8] J. E. Prussing, "Optimal two- and three-impulse fixed-time rendezvous in the vicinity of a circular orbit," AIAA Journal, vol. 8, pp. 1221-1228, 1970.

[9] J. E. Prussing and B. A. Conway, Orbital Mechanics, Oxford University Press, Oxford, UK, 1993.

[10] J. Tschauner and P. Hempel, "Rendez-vous with a target in an elliptical orbit," Astronautica Acta, vol. 11, no. 2, pp. 104-109, 1965.

[11] L. P. Abrahamson and R. G. Stern, "Two-body linear guidance matrices," Experimental Astronomy Laboratory Report RE-14, MIT, Cambridge, Mass, USA, 1965.

[12] J. L. Garrison, T. G. Gardner, and P. Axelrad, "Relative motion in highly elliptical orbits," Advances in the Astronautical Sciences, vol. 89, pp. 1359-1376, 1995.

[13] T. E. Carter, "State transition matrices for terminal rendezvous studies: brief survey and new example," Journal of Guidance, Control, and Dynamics, vol. 21, no. 1, pp. 148-155, 1998.

[14] R. G. Melton, "Time-explicit representation of relative motion between elliptical orbits," Journal of Guidance, Control, and Dynamics, vol. 23, no. 4, pp. 604-610, 2000.

[15] H. Schaub, "Relative orbit geometry through classical orbit element differences," Journal of Guidance, Control, and Dynamics, vol. 27, no. 5, pp. 839-848, 2004.

[16] H. Schaub and K. T. Alfriend, " $\mathrm{J}_{2}$ invariant relative orbits for spacecraft formations," Celestial Mechanics and Dynamical Astronomy, vol. 79, no. 2, pp. 77-95, 2001.

[17] K. T. Alfriend and H. Schaub, "Dynamic and control of spacecraft formations: challenges and some solutions," Journal of the Astronautical Sciences, vol. 48, no. 2-3, pp. 249-267, 2000.

[18] S. P. Hughes and C. D. Hall, "Optimal configurations for rotating spacecraft formations," Journal of the Astronautical Sciences, vol. 48 , no. 2-3, pp. 225-247, 2000.
[19] D. F. Chichka, "Satellite clusters with constant apparent distribution," Journal of Guidance, Control, and Dynamics, vol. 24, no. 1, pp. 117-122, 2001.

[20] G. Inalhan, M. Tillerson, and J. P. How, "Relative dynamics and control of spacecraft formations in eccentric orbits," Journal of Guidance, Control, and Dynamics, vol. 25, no. 1, pp. 48-59, 2002.

[21] S. R. Vadali, S. S. Vaddi, and K. T. Alfriend, "An intelligent control concept for formation flying satellites," International Journal of Robust and Nonlinear Control, vol. 12, no. 2-3, pp. $97-$ $115,2002$.

[22] S. S. Vaddi, S. R. Vadali, and K. T. Alfriend, "Formation flying: accommodating nonlinearity and eccentricity perturbations," Journal of Guidance, Control, and Dynamics, vol. 26, no. 2, pp. 214-223, 2003.

[23] J. K. Eyer and C. J. Damaren, "Quasi-periodic relative trajectory generation for formation flying satellites," Journal of Guidance, Control, and Dynamics, vol. 32, no. 4, pp. 1403-1406, 2009.

[24] T. E. Carter, "Fuel-optimal maneuvers of a spacecraft relative to a point in circular orbit," Journal of Guidance, Control, and Dynamics, vol. 7, no. 6, pp. 710-716, 1984.

[25] L. Breger and J. P. How, "Safe trajectories for autonomous rendezvous of spacecraft," Journal of Guidance, Control, and Dynamics, vol. 31, no. 5, pp. 1478-1489, 2008.

[26] D. J. Irvin, R. G. Cobb, and T. A. Lovell, "Fuel-optimal maneuvers for constrained relative satellite orbits," Journal of Guidance, Control, and Dynamics, vol. 32, no. 3, pp. 960-973, 2009.

[27] P. Lu and X. Liu, "Autonomous trajectory planning for rendezvous and proximity operations by conic optimization," Journal of Guidance, Control, and Dynamics, vol. 36, no. 2, pp. 375-389, 2013.

[28] W. E. Wiesel, "Optimal impulsive control of relative satellite motion," Journal of Guidance, Control, and Dynamics, vol. 26, no. 1, pp. 74-78, 2003.

[29] P. Gurfil, "Control-theoretic analysis of low-thrust orbital transfer using orbital elements," Journal of Guidance, Control, and Dynamics, vol. 26, no. 6, pp. 979-983, 2003.

[30] S. S. Vaddi, K. T. Alfriend, S. R. Vadali, and P. Sengupta, "Formation establishment and reconfiguration using impulsive control," Journal of Guidance, Control, and Dynamics, vol. 28, no. 2, pp. 262-268, 2005.

[31] M. Pontani and B. A. Conway, "Optimal low-thrust orbital maneuvers via indirect swarming method," Journal of Optimization Theory and Applications, vol. 162, no. 1, pp. 272-292, 2014.

[32] M. Pontani and B. A. Conway, "Optimal finite-thrust rendezvous trajectories found via particle swarm algorithm," Journal of Spacecraft and Rockets, vol. 50, no. 6, pp. 1222-1234, 2013.

[33] M. Pontani and B. A. Conway, "Minimum-fuel finite-thrust relative orbit maneuvers via indirect heuristic method," Journal of Guidance, Control, and Dynamics, vol. 38, no. 5, pp. 913-924, 2015.

[34] D. Hull, Optimal Control Theory for Applications, Springer, New York, NY, USA, 2003. 


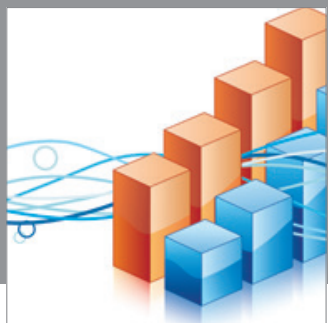

Advances in

Operations Research

mansans

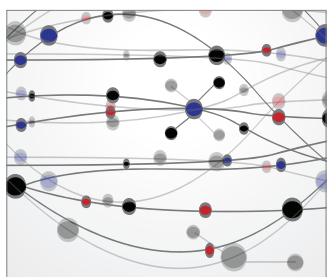

The Scientific World Journal
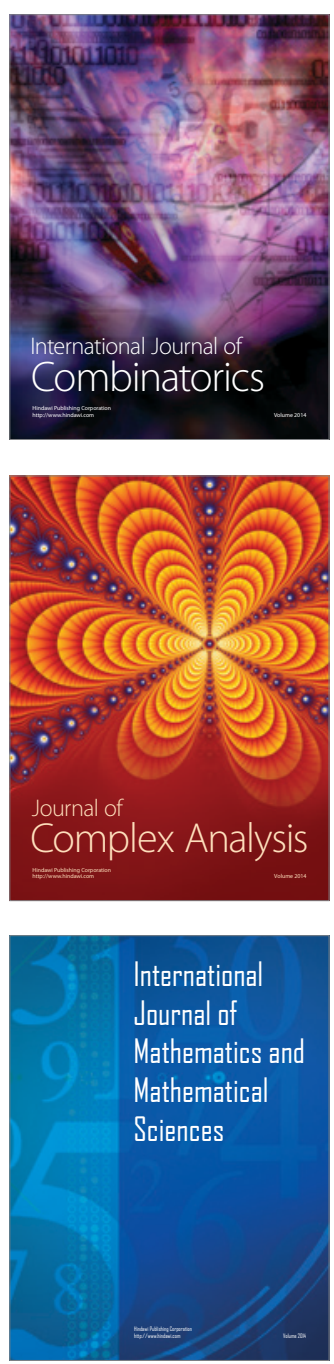
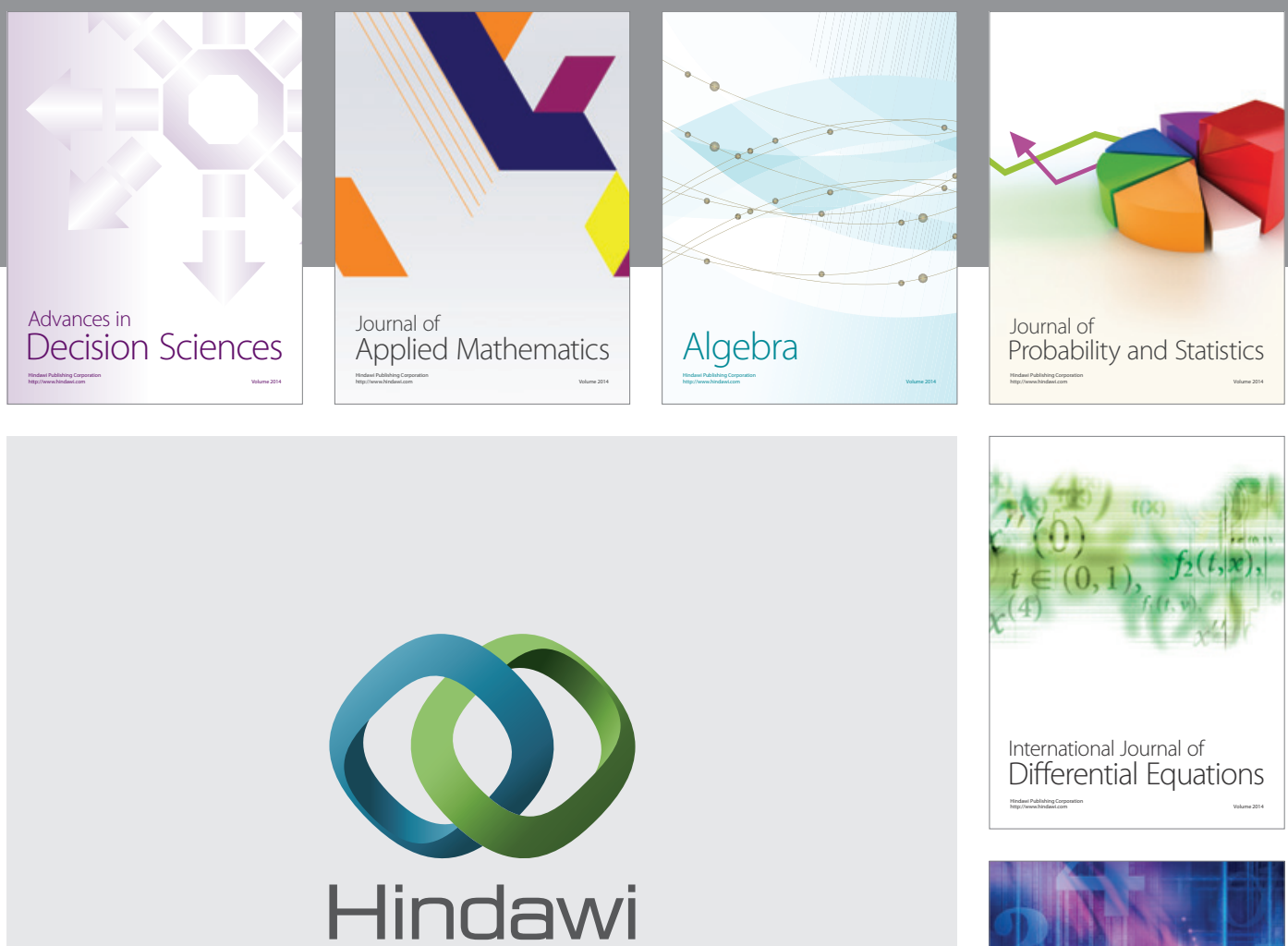

Submit your manuscripts at http://www.hindawi.com
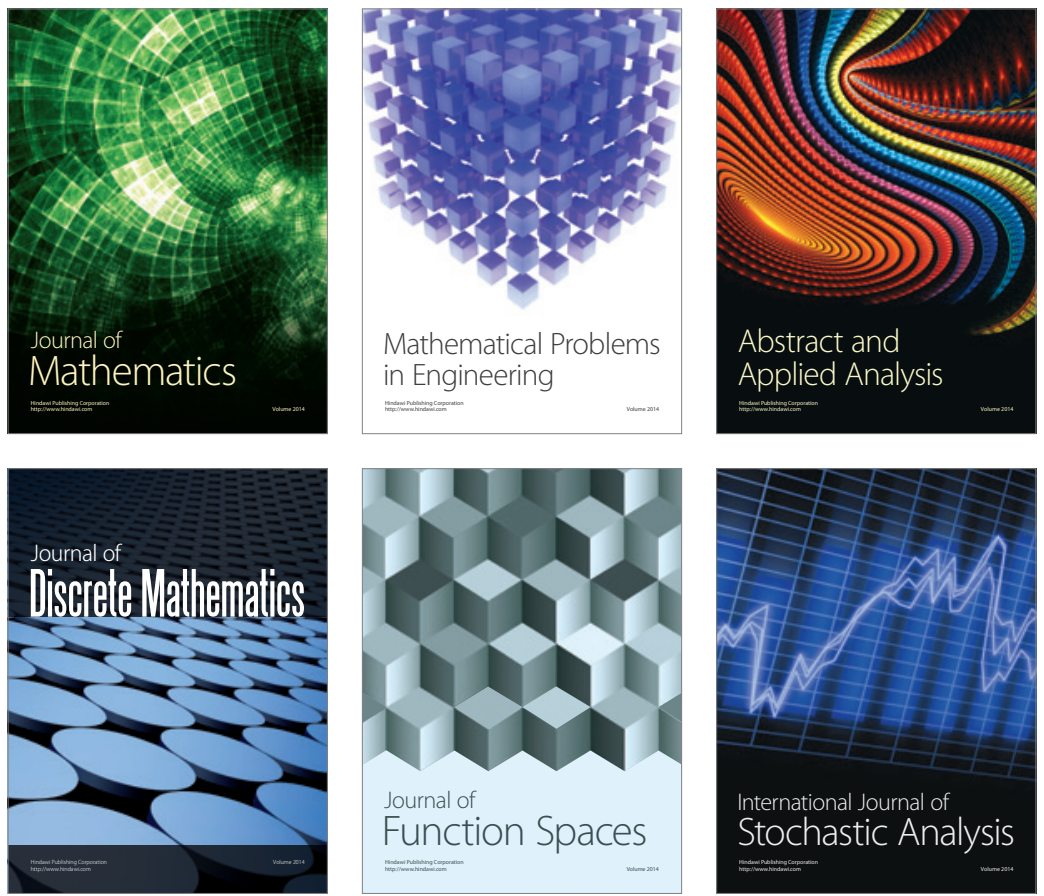

Journal of

Function Spaces

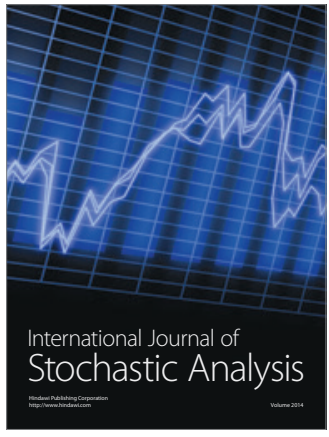

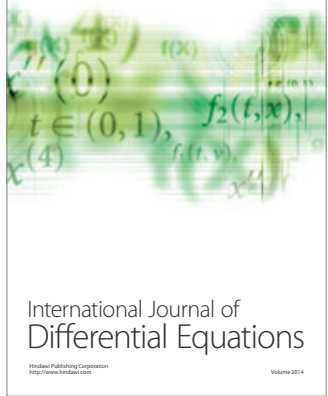
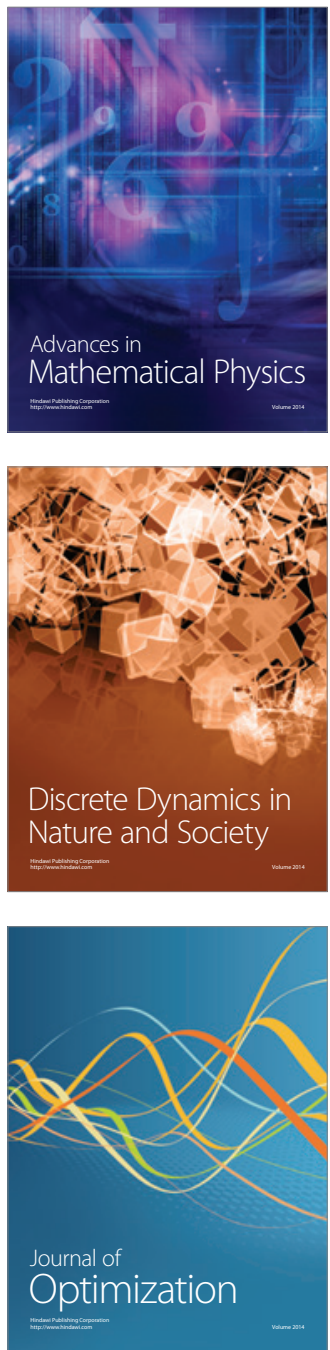